\title{
DISCRETE ANALOGUES OF CERTAIN INTEGRAL INEQUALITIES
}

\author{
H. D. BLOCK ${ }^{1}$
}

1. Introduction. In a previous paper [1] a class of inequalities was derived which bounded $\max _{x}|y(x)|$ in terms of integrals involving $y$ and $d y / d x$. In the present paper analogous results are found for the discrete case. In forming the analogue of the derivative there is some question as to what condition at the boundary is appropriate. In some situations it is appropriate to use the periodic end conditions; i.e., if $Z=\left(z_{1}, \cdots, z_{N}\right)$ then one takes $D Z=\left(z_{2}-z_{1}, z_{3}-z_{2}, \cdots\right.$, $\left.z_{N}-z_{N-1}, z_{1}-z_{N}\right)$ as the analogue of the derivative. In other situations it appears more natural to use an $(N-1)$-vector $\dot{Z}=\left(z_{2}-z_{1}, \cdots, z_{N}\right.$ $\left.-z_{N-1}\right)$. Each of these cases will be dealt with here; for $D Z$ we use a technique which is patterned after an eigenfunction technique in the continuous case and for $\dot{Z}$ a device which imitates the use of the Green's Function which we made in [1]. The author is indebted to Professors C. Herz and P. C. Rosenbloom for helpful discussions on techniques in the continuous case which suggested some of the methods used below.

2. Periodic end conditions. Let $z_{1}, z_{2}, \cdots, z_{N}$ be complex numbers, $N \geqq 3$. Then $Z=\left(z_{1}, \cdots, z_{N}\right)$ is a vector in unitary $N$-space, $U_{N}$, with the inner product $(Z, Y)=\sum_{n=1}^{N} z_{n} \bar{y}_{n}$. Let $H$ be an operator such that $H \Phi_{n}=\lambda_{n} \Phi_{n}, n=1, \cdots, N$, where $\left\{\Phi_{1}, \cdots, \Phi_{N}\right\}$ is an orthonormal basis for $U_{N}$. Now $Z=\sum_{n=1}^{N} c_{n} \Phi_{n}$, where $c_{n}=\left(Z, \Phi_{n}\right) ;\|Z\|^{2}$ $=\sum_{n=1}^{N}\left|c_{n}\right|^{2}$ and $\|H Z\|^{2}=\sum_{n=1}^{N}\left|\lambda_{n} c_{n}\right|^{2}$. Let $\Phi_{n}=\left(\phi_{1}(n), \phi_{2}(n), \cdots\right.$, $\left.\phi_{N}(n)\right)$.

Let the integers $(1,2, \cdots, N)$ be divided into two disjoint sets $I$ and $J$ and let $d$ be a number such that $d \neq \lambda_{j}$ for any $j$ in $J$. Then for any $k$ in $I \cup J$

$$
\begin{aligned}
\boldsymbol{z}_{\boldsymbol{k}} & =\sum_{n=1}^{N} c_{n} \phi_{k}(n) \\
& =\sum_{i \in I} c_{i} \phi_{k}(i)+d \sum_{j \in J} \frac{c_{j} \phi_{k}(j)}{d-\lambda_{j}}-\sum_{j \in J} \frac{\lambda_{j} c_{j} \phi_{k}(j)}{d-\lambda_{j}} .
\end{aligned}
$$

Hence by Schwarz's Inequality

Received by the editors January 16, 1957.

1 Research under contract with the Office of Naval Research. 


$$
\begin{aligned}
\left|z_{k}\right| \leqq & \left(\sum_{i \in I}\left|c_{i}\right|^{2}\right)^{1 / 2}\left(\sum_{i \in I}\left|\phi_{k}(i)\right|^{2}\right)^{1 / 2} \\
& +|d|\left(\sum_{j \in J}\left|c_{j}\right|^{2}\right)^{1 / 2}\left(\sum_{j \in J}\left|\frac{\phi_{k}(j)}{d-\lambda_{j}}\right|^{2}\right)^{1 / 2} \\
& +\left(\sum_{j \in J}\left|\lambda_{j} c_{j}\right|^{2}\right)^{1 / 2}\left(\sum_{j \in J}\left|\frac{\phi_{k}(j)}{d-\lambda_{j}}\right|^{2}\right)^{1 / 2} .
\end{aligned}
$$

Thus

$$
\left|z_{k}\right| \leqq\left(A_{k}+|d| B_{k}\right)\|Z\|+B_{k}\|H Z\|,
$$

where the quantities

$$
A_{k}=\left(\sum_{i \in I}\left|\phi_{k}(i)\right|^{2}\right)^{1 / 2}, \quad B_{k}=\left(\sum_{j \in J}\left|\frac{\phi_{k}(j)}{d-\lambda_{j}}\right|^{2}\right)^{1 / 2}
$$

do not depend on the particular vector $Z$.

To illustrate (2) let $H Z=D Z=\left(z_{2}-z_{1}, \cdots, z_{1}-z_{N}\right)$. Then

$$
\begin{aligned}
\left.\left.\phi_{k}\right) n\right) & =\frac{e^{2 \pi i n k / N}}{N^{1 / 2}}, \\
\lambda_{n} & =e^{2 \pi i n / N}-1 ;
\end{aligned}
$$

let $d=0$ and let $I=(N)$. Then

$$
\begin{aligned}
A_{k} & =\frac{1}{N^{1 / 2}}, \\
B_{k} & =\frac{1}{2 N^{1 / 2}}\left(\sum_{j=1}^{N-1} \csc ^{2} \pi j / N\right)^{1 / 2} \leqq \frac{1}{2}\left(\frac{N-1}{N}\right)^{1 / 2} \csc \frac{\pi}{N} .
\end{aligned}
$$

Hence we have

TheOREM 1. For any $Z=\left(z_{1}, \cdots, z_{N}\right)$

$$
\sup _{1 \leqq k \leqq N}\left|z_{k}\right| \leqq \frac{1}{N^{1 / 2}}\|Z\|+\frac{1}{2}(\csc \pi / N)\|D Z\| .
$$

The equality holds if and only if $Z=C(1,1, \cdots, 1)$.

If $Z$ satisfies special conditions, such as $\sum_{n=1}^{N} z_{n}=0$, then discrete analogues of Wirtinger's Inequality can be obtained, using the notation of the above illustration, as follows. Since $c_{N}=\left(Z, \Phi_{N}\right)$ $=N^{-1 / 2} \sum_{n=1}^{N} z_{n}$, the condition $\sum_{n=1}^{N} z_{n}=0$ is equivalent to $c_{N}=0$. Then 


$$
\begin{aligned}
\|D Z\|^{2} & =\sum_{n=1}^{N}\left|c_{n} \lambda_{n}\right|^{2}=\sum_{n=1}^{N} 4\left|c_{n}\right|^{2} \sin ^{2} \frac{\pi n}{N}=\sum_{n=1}^{N-1} 4\left|c_{n}\right|^{2} \sin ^{2} \frac{\pi n}{N} \\
& \geqq 4 \sin ^{2} \frac{\pi}{N} \sum_{n=1}^{N}\left|c_{n}\right|^{2}=4 \sin ^{2} \frac{\pi}{N}\|Z\|^{2} .
\end{aligned}
$$

The equality holds if and only if $Z$ is a linear combination of $\Phi_{1}$ and $\Phi_{N-1}$, or, equivalently, of $(\sin (2 \pi / N)$, $\sin (4 \pi / N), \cdots, \sin 2 \pi)$ and $(\cos (2 \pi / N), \cdots, \cos 2 \pi)$.

To deal with the case where it is given instead that $z_{N}=0$, define $z_{N+k}=-z_{k}(k=1, \cdots, N)$ and apply the result just obtained to $\left(z_{1}, \cdots, z_{2 N}\right)$, since $\sum_{n=1}^{2 N} z_{n}=0$. From $z_{N}=0$ it follows that $\|Z\|^{2}$ and $\|D Z\|^{2}$ are just doubled in the new computation. Hence $\|D Z\|$ $\geqq 2 \sin (\pi / 2 N)\|Z\|$; the equality will hold for such $Z$ if and only if $z_{k}=C \sin (\pi k / N)$, since the condition $z \pi=0$ must now also be satisfied.

Since any vector of the form $Y=D Z=\left(y_{1}, \cdots, y_{N}\right)$ has $\sum_{n=1}^{N} y_{n}$ $=0$ we have $\left\|D^{k+1} Z\right\| \geqq(2 \sin (\pi / N))^{k}\|D Z\|$; if $\sum_{n=1}^{N} z_{n}=0$ this gives $\left\|D^{k} Z\right\| \geqq(2 \sin (\pi / N))^{k}\|Z\|$, which is achieved if $Z$ is a linear combination of $\Phi_{1}$ and $\Phi_{N-1}$. If, instead, $z_{N}=0$ then $\left\|D^{2} Z\right\| \geqq 2 \sin (\pi / N)\|D Z\|$ $\geqq 4 \sin (\pi / N) \sin (\pi / 2 N)\|Z\|$, but the equality here cannot be achieved unless $Z=0$.

Clearly the same inequalities hold if one uses backward instead of forward differences and, for the higher orders, any mixture.

Similar results can be obtained for several dimensions by an analogous method. For example, with a two-dimensional array of $M$ columns and $N$ rows $Z=z_{m n}, m=1, \cdots, M ; n=1, \cdots, N$, taking $\left(\Phi_{m n}\right)$ as a normal orthogonal system, where $\left(\Phi_{m n}\right)_{r s}$ $=\left(1 /(M N)^{1 / 2}\right) e^{2 \pi i(m r / M+n s / N)}$, one finds in the same manner that if $\sum_{n=1}^{N} \sum_{m=1}^{M} z_{m n}=0$, then

$$
\left\|D_{x} Z\right\|^{2}+\left\|D_{y} Z\right\|^{2} \geqq[2 \min (\sin (\pi / M), \sin (\pi / N))\|Z\|]^{2}
$$

and

$$
\left\|\left(D_{x}^{2}+D_{y}^{2}\right) Z\right\| \geqq[2 \min (\sin (\pi / M), \sin (\pi / N))]^{2}\|Z\|,
$$

with equality holding for each of these for a suitable choice of $Z$; indeed $Z$ may be chosen with real components, so that the inequality is sharp in the real case also. Similarly, if the numbers $z_{m n}$ vanish on two adjacent edges of the rectangle, i.e., $z_{n N}=z_{M n}=0$, then

$$
\left\|D_{x} Z\right\|^{2}+\left\|D_{y} Z\right\|^{2} \geqq 4\left[\sin ^{2}(\pi / 2 M)+\sin ^{2}(\pi / 2 N)\right]\|Z\|^{2}
$$

and 
$\left\|D_{x}^{2} Z+D_{y}^{2} Z\right\| \geqq 4(\sin (\pi / M) \sin (\pi / 2 M)+\sin (\pi / N) \sin (\pi / 2 N))\|Z\|^{2}$, where the first equality is achievable, but the second is not. All of this may be summed up in the following theorem, some of which is already contained in [2] where the proof is slightly more laborious.

THEOREM 2.

(a) If $\sum_{n=1}^{N} z_{n}=0$, then $\|D Z\| \geqq(2 \sin (\pi / N))\|Z\|$,

(b) if $z_{N}=0$, then $\|D Z\| \geqq(2 \sin (\pi / 2 N))\|Z\|$,

(c) $\left\|D^{k+1} Z\right\| \geqq(2 \sin (\pi / N))^{k}\|D Z\|$,

(d) if $\sum_{n=1}^{N} z_{n}=0$, then $\left\|D^{k} Z\right\| \geqq(2 \sin (\pi / N))^{k}\|Z\|$,

(e) if $\sum_{m=1}^{M} \sum_{n=1}^{N} z_{n m}=0$, then

$$
\begin{aligned}
&\left\|D_{x} Z\right\|^{2}+\left\|D_{y} Z\right\|^{2} \geqq[2 \min (\sin (\pi / M), \sin (\pi / N))\|Z\|]^{2} \text { and } \\
&\left\|D_{x}^{2} Z+D_{y}^{2} Z\right\| \geqq[2 \min (\sin (\pi / M), \sin (\pi / N))]^{2}\|Z\|,
\end{aligned}
$$

(f) if $z_{m N}=z_{M_{n}}=0,(m=1, \cdots, M ; n=1, \cdots, N)$ then $\left\|D_{x} Z\right\|^{2}$ $+\left\|D_{y} Z\right\|^{2} \geqq 4\left[\sin ^{2}(\pi / 2 M)+\sin ^{2}(\pi / 2 N)\right]\|Z\|^{2}$.

(g) if $z_{N}=0$ then $\left\|D^{2} Z\right\| \geqq 4 \sin (\pi / N) \sin (\pi / 2 N)\|Z\|$,

(h) if $\boldsymbol{z}_{m N}=\boldsymbol{z}_{M n}=0(m=1, \cdots, M ; n=1, \cdots, N)$, then

$$
\left\|D_{x}^{2} Z+D_{y}^{2} Z\right\| \geqq 4\left(\sin \frac{\pi}{M} \sin \frac{\pi}{2 M}+\sin \frac{\pi}{N} \sin \frac{\pi}{2 N}\right)\|Z\|^{2},
$$

where the equalities (a)-(f) can be achieved for nonzero real vectors as given in the text, but $(\mathrm{g})$ and $(\mathrm{h})$ can not.

REMARK. A case in which it is reasonable to lower the dimension by $t w o$ is when we seek the minimum of $\left\|\Delta^{2} Z\right\|$ subject to $z_{1}=z_{N}=0$, and take $\Delta^{2}\left(0, z_{2}, \cdots, z_{N-1}, 0\right)=\left(z_{3}-2 z_{2}, z_{4}-2 z_{3}+z_{2}, \cdots, z_{N-1}\right.$ $\left.-2 z_{n-2}+z_{N-3},-2 z_{N-1}+z_{N-2}\right)$. Then $\Theta_{n}=(2 /(N-2))^{1 / 2}(0$, $\sin (2 n \pi /(N-1)), \sin (4 n \pi /(N-1)), \cdots, \sin (2(N-2) n \pi /(N-1))$, $0), n=1,2, \cdots, N-2$ form an orthonormal basis in the subspace of interest with $\Delta^{2} \Theta_{n}=-4(\sin \quad(n \pi /(N-1)))^{2}(2 /(N-1))^{1 / 2}$ $(\sin (2 n \pi /(N-1)), \sin (4 n \pi /(N-1)), \cdots, \sin (2(N-2) n \pi /(N-1)))$. Thus if $z_{1}=z_{N}=0$ it readily follows that $\left\|\Delta^{2} Z\right\| \geqq 4 \sin ^{2}(\pi /(N-1))$ $\|z\|$.

3. Reduction of dimension. Here we define $\dot{Z}$ as the $(n-1)$ vector $\left(z_{2}-z_{1}, z_{3}-z_{2}, \cdots, z_{N}-z_{N-1}\right)=\left(\dot{z}_{1}, \dot{z}_{2}, \cdots, \dot{z}_{N-1}\right)$ with the corresponding inner product

$$
\sum_{i=1}^{N-1} \dot{z}_{i} \dot{y}_{i}=(\dot{Z}, \dot{Y})
$$


For a fixed $k$ such that $1 \leqq k \leqq N$ suppose that $W=W(k)=\left(w_{1}(k)\right.$, . . , $\left.w_{N}(k)\right)$ satisfies: $(L \dot{W}, M \dot{Y})+(R W, S Y)=y_{k}$ for all $Y$ $=\left(y_{1}, \cdots, y_{N}\right)$ in a subset $V$ of $U_{N}$, where $L$ and $M$ are operators on $U_{N-1}$ and $R$ and $S$ are operators on $U_{N}$. Then

$$
\begin{aligned}
& \left|y_{k}\right| \leqq\|L \dot{W}\| \cdot\|M \dot{Y}\|+\|R W\| \cdot\|S Y\|, \\
& \left|y_{k}\right|^{2} \leqq\left(\|L \dot{W}\|^{2}+\|R W\|^{2}\right)\left(\|M \dot{Y}\|^{2}+\|S Y\|^{2}\right),
\end{aligned}
$$

or

$$
\left|y_{k}\right|^{2} \leqq B^{2}(k)\left(\|M \dot{Y}\|^{2}+\|S Y\|^{2}\right),
$$

where $B^{2}(k)=\|L \dot{W}\|^{2}+\|R W\|^{2}$ does not depend on the particular vector $Y$. If $L=M$ and $R=S$, then, if $W \in V$, we may take $Y=W$ so that $B^{2}(k)=w_{k}(k)$, which simplifies some computations. We illustrate the technique for the case when $L=M=$ the identity operator and $R=S=$ a positive constant $c$ times the identity. The results above can then be summarized as follows. If $W=W(k)$ satisfies

$$
(\dot{W}, \dot{Y})+c^{2}(W, Y)=y_{k}
$$

for all $Y$ in $V \subseteq U_{N}$, then for all $Y$ in $V$

$$
\left|y_{k}\right|^{2} \leqq\left(\|\dot{W}\|^{2}+c^{2}\|W\|^{2}\right)\left(\|\dot{Y}\|^{2}+c^{2}\|Y\|^{2}\right) \text {. }
$$

Let $\lambda$ be a positive number $\neq 1$, let $c^{2}=\lambda+1 / \lambda-2$ and let $a=\log \lambda$. Let $k$ be fixed $(1 \leqq k \leqq N)$; let $W(k)=\left(w_{1}, w_{2}, \cdots, w_{N}\right)$ where

$$
\begin{array}{ll}
w_{i}=A \lambda^{i}+B \lambda^{-i} & \text { for } 1 \leqq i \leqq k, \\
w_{j}=C \lambda^{i}+D \lambda^{-i} & \text { for } k \leqq j \leqq N .
\end{array}
$$

This implies that we are taking

$$
D-B=\lambda^{2 k}(A-C) \text {. }
$$

Then

$$
\begin{aligned}
\sum_{i=1}^{N-1} \dot{w}_{i} \dot{y}_{i}= & \sum_{i=1}^{N-1}\left(w_{i+1}-w_{i}\right) y_{i+1}-\sum_{i=0}^{N-2}\left(w_{i+2}-w_{i+1}\right) y_{i+1} \\
= & -\sum_{i=2}^{N-1}\left(w_{i+1}-2 w_{i}+w_{i-1}\right) y_{i}+\left(w_{N}-w_{N-1}\right) y_{N}-\left(w_{2}-w_{1}\right) y_{1} \\
= & -\sum_{i=1}^{N} c^{2} w_{i} y_{i}+\left\{c^{2} w_{k} y_{k}-\left(w_{(k+1)}-2 w_{k}+w_{k-1}\right) y_{k}\right\} \\
& +\left[w_{N}\left(1+c^{2}\right)-w_{N-1}\right] y_{N}+\left[w_{1}\left(1+c^{2}\right)-w_{2}\right] y_{1},
\end{aligned}
$$

where the term in \{\} is to be set equal to zero if $k=1$ or $k=N$. 


\section{If $1<k<N$}

$$
\begin{aligned}
& (\dot{W}, \dot{Y})+c^{2}(W, Y) \\
& \quad=y_{k}+(\lambda-1)\left[\left(C \lambda^{N}-D \lambda^{-N-1}\right) y_{N}+\left(B \lambda^{-1}-A\right) y_{1}\right]
\end{aligned}
$$

provided that

$$
(A-C) \lambda^{k+1}+(B-D) \lambda^{-k-1}=1 .
$$

For $k=1$

$$
\begin{aligned}
(\dot{W}, \dot{Y})+c^{2}(W, Y) & =\left[w_{N}\left(1+c^{2}\right)-w_{N-1}\right] y_{N}+\left[w_{1}\left(1+c^{2}\right)-w_{2}\right\rfloor y_{1} \\
& =y_{1}+(\lambda-1)\left(C \lambda^{N}-D \lambda^{-N-1}\right) y_{N}
\end{aligned}
$$

provided that

$$
(\lambda-1)(D-\lambda C)=\lambda
$$

and for $k=N$ we get

$$
(\dot{W}, \dot{Y})+c^{2}(W, Y)=y_{N}+\lambda^{-1}(1-\lambda)[A \lambda-B] y_{1}
$$

provided that

$$
(\lambda-1)\left(A \lambda^{N}-B \lambda^{-N-1}\right)=1 .
$$

Now we get several types of inequality (5) according to class of vectors $Y$ is permitted to belong to. We consider three cases: (I) $Y$ unrestricted, (II) $y_{1}=K y_{N}$ where $K$ is a fixed constant, (III) $y_{1}=y_{N}$ $=0$. Naturally we expect smaller bounds as we go from (I) to (II) to (III).

CASE I. For $1<k<N$ we require that $A, B, C, D$ satisfy (6), (8), and, in order that (7) be of the form (4) for all $Y$ in $U_{N}$ :

$$
\begin{aligned}
C \lambda^{N} & =D \lambda^{-N-1}, \\
B & =\lambda A .
\end{aligned}
$$

Then

$$
\|\dot{W}\|^{2}+c^{2}\|W\|^{2}=w_{k}(k)=\frac{\lambda^{2 N+1}+\lambda^{2 k}+\lambda^{2(N-k+1)}+\lambda}{\left(\lambda^{2}-1\right)\left(\lambda^{2 N}-1\right)} .
$$

For $k=1$, we require (6), (8a) and $D=C \lambda^{2 N+1}$. These guarantee that (7a) reduces to the form (4); then $w_{1}(1)=\left(\lambda+\lambda^{2 N}\right) /(\lambda-1)\left(\lambda^{2 N}-1\right)$. Similarly for $k=N$ we take (6), (8b) and $B=\lambda A$ so that (7b) reduces to the form (4). Then $w_{N}(N)=\left(\lambda^{N}+\lambda^{-N+1}\right) /(\lambda-1)\left(\lambda^{N}-\lambda^{-N}\right)$. Hence we have

THEOREM 3. For any set of numbers $y_{1}, \cdots, y_{N}$; and any $\lambda, 0 \neq \lambda \neq 1$, 


$$
\begin{aligned}
\left|y_{k}\right|^{2} \leqq & \frac{\left(\lambda^{2 N+1}+\lambda^{2 k}+\lambda^{2(N-k+1)}+\lambda\right)}{\left(\lambda^{2}-1\right)\left(\lambda^{2 N}-1\right)} \\
& \cdot\left(\|\dot{Y}\|^{2}+\left(\lambda^{1 / 2}-1 / \lambda^{1 / 2}\right)^{2}\|Y\|^{2}\right)
\end{aligned}
$$

or, letting $\lambda=e^{a}$,

$$
\begin{aligned}
&\left|y_{k}\right|^{2} \leqq \frac{\cosh a N+\cosh a(N-2 k+1)}{2 \sinh a \sinh a N} \\
& \cdot\left(\|\dot{Y}\|^{2}+2(\cosh a-1)\|Y\|^{2}\right)
\end{aligned}
$$

in particular the uniform bound:

$$
\begin{aligned}
&\left|y_{k}\right|^{2} \leqq \frac{1}{2}[\operatorname{coth} a N(\operatorname{csch} a+\operatorname{coth} a)-1] \\
& \cdot\left(\|\dot{Y}\|^{2}+2(\cosh a-1)\|Y\|^{2}\right) .
\end{aligned}
$$

The equalities in (11) and (12) are achieved if $Y=W(k)$ and, with $k=1$, equality is also achieved in (13).

CASE II. If $1<k<N$ then we require (6), (8) and $C \lambda^{N}-D \lambda^{-N-1}$ $+\left(B \lambda^{-1}-A\right) K=0$, in order that (7) be of the form (4). Then from (7) with $Y$ replaced by $W$ we get

$$
\begin{aligned}
& \|\dot{W}\|^{2}+c^{2}\|W\|^{2}=w_{k}+(\lambda-1)\left(B \lambda^{-1}-A\right)\left[w_{1}-K w_{N}\right] \\
& \quad=A \lambda^{k}+B \lambda^{-k}+(\lambda-1)\left(B \lambda^{-1}-A\right)\left[A \lambda+B \lambda^{-1}-K C \lambda^{N}-K D \lambda^{-N}\right]
\end{aligned}
$$

and one can minimize this with respect to the free parameter. For example with $K=0$ we get for $1<k<N$

$$
\|\dot{W}\|^{2}+c^{2}\|W\|^{2}=\frac{\cosh a(N+1 / 2-k) \sinh a(k-1)}{\cosh a(N-1 / 2) \sinh a} .
$$

For $k=N$ we require (6) and (8b) since the condition $y_{1}=0$ then guarantees that $(7 \mathrm{~b})$ is of the form (4). Then from (7b) with $Y$ replaced by $W$ we get:

$\|\dot{W}\|^{2}+c^{2}\|W\|^{2}=A \lambda^{N}+B \lambda^{-N}+\lambda^{-1}(1-\lambda)(A \lambda-B)\left(A \lambda+B \lambda^{-1}\right)$, which, when we minimize with respect to the free parameter, gives the same form as above, so that we get the following theorem

Theorem 4. Let $y_{1}, y_{2}, \cdots, y_{N}$ be such that $y_{1}=0$; then for $1 \leqq k \leqq N$

$$
\left|y_{k}\right|^{2} \leqq \frac{\cosh (a(N-k+1 / 2)) \sinh (a(k-1))\left(\|\dot{Y}\|^{2}+c^{2}\|Y\|^{2}\right)}{\cosh (a(N-1 / 2)) \sinh a},
$$

where $a=\left|\operatorname{arc} \cosh \left(c^{2} / 2+1\right)\right|$. 
In particular, taking the maximum:

$$
\left|y_{k}\right|^{2} \leqq \frac{\cosh (a / 2) \sinh a(N-1)}{\cosh a(N-1 / 2) \sinh a}\left(\|\dot{Y}\|^{2}+c^{2}\|Y\|^{2}\right)
$$

or, summing (14):

$$
\begin{aligned}
& \sum_{k=1}^{N}\left|y_{k}\right|^{2} \\
& \leqq \frac{(N \sinh (a(N-1 / 2)) \sinh a-\sinh N a \sinh (a / 2))\left(\|\dot{Y}\|^{2}+c^{2}\|Y\|^{2}\right)}{2 \cosh a(N-1 / 2) \sinh ^{2} a} .
\end{aligned}
$$

CASE III. Now we have only (6) and (8) as constraints with $\|\dot{W}\|^{2}$ $+c^{2}\|W\|^{2}$ given by (7); minimizing this with respect to the two free parameters we get

TheOREM 5. Let $y_{1}, \cdots, y_{N}$ be such that $y_{1}=y_{N}=0$; then for $0 \leqq k \leqq N$

$$
\left|y_{k}\right|^{2} \leqq \frac{\sinh (k-1) a \sinh (N-k) a}{\sinh a \sinh (N-1) a}\left(\|\dot{Y}\|^{2}+c^{2}\|Y\|^{2}\right),
$$

where $a=\left|\operatorname{arc} \cosh \left(c^{2} / 2+1\right)\right|$.

In particular, taking the maximum of the right side:

$$
\left|y_{k}\right|^{2} \leqq \frac{\tanh ((N-1) / 2) a}{2 \sinh a}\left(\|\dot{Y}\|^{2}+c^{2}\|Y\|^{2}\right) ;
$$

or, summing (16):

$$
\sum_{k=1}^{N}\left|y_{k}\right|^{2} \leqq\left[\frac{(N-1) \operatorname{coth}(N-1) a-\operatorname{coth} a}{2 \sinh a}\right]\left(\|\dot{Y}\|^{2}+c^{2}\|Y\|^{2}\right) .
$$

1. H. D. Block, $A$ class of inequalities, Proc. Amer. Math. Soc. vol. 8 (1957) pp. 844-851.

2. K. Fan, O. Taussky and J. Todd, Discrete analogs of inequalities of Wirtinger, Monatshefte für Mathematik vol. 59 (1955) pp. 73-90.

3. Hardy, Littlewood and Polya, Inequalities, Cambridge University Press, 1934.

Cornell University 